\title{
INDIRECT LOCALLY INTERNAL OBSERVABILITY AND CONTROLLABILITY OF WEAKLY COUPLED WAVE EQUATIONS
}

\author{
Ali Wehbe And WAEL YousseF
}

\begin{abstract}
In this paper, we study the exact controllability of a system of weakly coupled wave equations with an internal locally control acted on only one equation. Using a piecewise multiplier method, we show that, for a sufficiently large time $T$, the observation of the velocity of the first component of the solution on a neighborhood of a part of the boundary allows us to get back a weakened energy of initial data of the second component of the solution, this if the coupling parameter is sufficiently small, but non vanishing. This result leads, by the HUM method, to prove that the total system is exactly controllable by means of one locally distributed control.
\end{abstract}

Mathematics subject classification (2010): 35B37, 35D05, 73K50, 93C20.

Keywords and phrases: Wave equation, coupled system, indirect observability, indirect exact controllability.

\section{REFERENCES}

[1] F. Alabau-Boussouira, A two-level energy method for indirect boundary observability and controllability of weakly coupled hyperbolic systems, SIAM J. Control Optim., 42 (2003), 871-906.

[2] F. Alabau-Boussouira, Obesrvabilité frontière indirecte de systèmes faiblement couplés, C. R. Acad. Sci. Paris Sér. I Math., 333 (2001), 645-650.

[3] F. Alabau-Boussouira, Indirect boundary stabilization of weakly coupled hyperbolic systems, SIAM J. Control Optim., 41 (2002), 511-541.

[4] F. Alabau, P. CANNARSA, AND V. KOMORNiK, Indirect internal stabilization of weakly coupled systems, J. Evol. Equ., 2 (2002), 127-150.

[5] F. Ammar-Khodjar, A. Benabdallah, C. DupaiX and I. Kostin, Null-controllability of some systems of parabolic type by one control force, ESAIM Control Optim. Calc. Var., 11 (2005), 426-448.

[6] F. Ammar-Khodjar, A. Benabdallah AND C. Dupaix, Null-controllability of some reactiondiffusion systems with one control force, J. Math. Anal. Appl., 320 (2006), 928-943.

[7] A. BEYRATH, Indirect internal stabilization of weakly coupled systems with locally distributed damping, C. R. Acad. Sci. Paris Sér. I Math., 333 (2001), 451-456.

[8] H. BrezIS, Analyse Fonctionelle, Théorie et Applications, Masson, Paris, 1992.

[9] F. CONRAD AND B. RAO, Decay of solutions of wave equations in a star-shaped domain with nonlinear boundary feedback, Asymptotic Anal., 7 (1993), 159-177.

[10] T. DUYCKAERTS, Optimal decay rates of the energy of a hyperbolic-parabolic system coupled by an interface, Asymptot. Anal., 51 (2007), 17-45.

[11] M. GonZÁLEZ-Burgos AND R. PÉREZ-GARCíA, Controllability results for some nonlinear coupled parabolic systems by one control force, Asymptot. Anal., 46 (2006), 123-162.

[12] V. KOMORNik, Exact Controllability and Stabilization, Masson, Paris, 1994.

[13] V. KOMORNIK, Rapid boundary stabilization of linear distributed systems, SIAM J. Control Optim., 35 (1997), 1591-1613.

[14] V. KOMORNIK AND P. LORETI, Ingham-type theorems for vector-valued functions and observability of coupled linear systems, SIAM J. Control Optim., 37 (1998), 461-485.

[15] I. LASIECKA AND R. TRIgGiani, Carleman estimates and exact boundary controllability for a system of coupled, nonconservative second-order hyperbolic equations, Paritial differential equation methodes in control and shape analysis, Lecture Notes in Pure and Appl. Math., 188 (1997), 215-243. 
[16] J. L. Lions, Contrôlabilité exacte, perturbations et stabilisation de systèmes distribués, Masson, Paris, 1988.

[17] J.L. LIONS, Exact controllability, stabilization and perturbations for distributed systems, SIAM Rev., 30 (1988), 1-68.

[18] J.L. Lions and E.Magenes, Problemes aux Limites Non-Homogenes et Applications, Vol. 1, Dunod,Paris, 1968.

[19] K. LIU, Locally distributed control and damping for the conservative systems, SIAM J. Control Optim., 35 (1997), 1574-1590.

[20] Z. LIU AND B. RAO, A spectral approach to the indirect boundary control of a system of weakly coupled wave equations, Discrete Contin. Dyn. Syst., 23 (2009), 399-414.

[21] Z. LIU AND B. RAO, Frequency domain approach for the polynomial stability of a system of partially damped wave equations, J. Math. Anal. Appl., 335 (2007), 860-881.

[22] P. LORETI AND B. RAO, Optimal energy decay rate for partially damped systems by spectral compensation, SIAM J. Control Optim., 45 (2006), 1612-1632.

[23] P. MARTinez, A new method to obtain decay rate estimates for dissipative systems with localized damping, Rev. Mat. Complut., 12 (1999), 251-283.

[24] A. PAZY, Semigroups of Linear Operators and Applications to Partial Differential Equations, Applied Mathematical Sciences, 44, Springer-Verlag, 1983.

[25] R. RAJARAM, M. NAJAFI, Exact controllability of wave equations in $\mathbb{R}^{n}$ coupled in parallel, J. Math. Anal. Appl., 356 (2009), 7-12.

[26] D. L. RuSSELL, A General framework for the study of indirect damping mechanisms in elastic systems, J. Math. Anal. Appl., 173 (1993), 339-358.

[27] D. RUSSELL, Decay rates for weakly damped systems in Hilbert space obtained with control- theoretic methods, J. Differential Equations, 19, 2 (1975), 344-370.

[28] A. Soufyane And A. Wenbe, Uniform stabilization for the Timoshenko beam by a locally distributed damping, Electron. J. Differential Equations, 29 (2003), 1-14.

[29] A. Wehbe, W. Youssef, Observabilité et controlabilité exacte indirecte interne par un controle localement distribué de systemes d'equations couplées, C. R. Math. Acad. Sci. Paris, 348 (2010), $1169-1173$.

[30] A. Wehbe And W. Youssef, Stabilization of the uniform Timoshenko beam by one locally distributed feedback, Appl. Anal., 88, 7 (2009), 1067-1078.

[31] A. WeHBe AND W. Youssef, Exponential and polynomial stability of an elastic Bresse system with two locally distributed feedback, J. Math. Phys., 51, 9 (2010), 17 pp.

[32] X. Zhang And E. Zuazua, Polynomial decay and control of a 1-d hyperbolic-parabolic coupled system, J. Differential Equations, 204 (2004), 380-438. 